\title{
T2 lesion location really matters: a 10 year follow-up study in primary progressive multiple sclerosis
}

\author{
B Bodini, ${ }^{1,2}$ M Battaglini, ${ }^{3}$ N De Stefano, ${ }^{3}$ Z Khaleeli, ${ }^{1}$ F Barkhof, ${ }^{4}$ D Chard, ${ }^{5}$ \\ M Filippi, ${ }^{6}$ X Montalban, ${ }^{7}$ C Polman, ${ }^{4}$ M Rovaris, ${ }^{6,8}$ A Rovira, ${ }^{7}$ R Samson, ${ }^{5}$ D Miller, ${ }^{5}$ \\ A Thompson, ${ }^{1} 0$ Ciccarelli ${ }^{1}$
}

\begin{abstract}
- An additional table 1 is published online only. To view this file please visit the journal online (http://jnnp.bmj.com).
\end{abstract}

${ }^{1}$ Department of Brain Repair and Rehabilitation, Institute of Neurology, University College London, London, UK ${ }^{2}$ Department of Neurological Sciences, University of Rome "La Sapienza", Rome, Italy ${ }^{3}$ Department of Neurological and Behavioural Sciences, University of Siena, Siena, Italy ${ }^{4}$ Department of Radiology and Department of Neurology, VU University Medical Center, Amsterdam, The Netherlands Department of Neuroinflammation, Institute of Neurology, University College London, London, UK ${ }^{6}$ Neuroimaging Research Unit Institute of Experimental Neurology, Division of Neuroscience, Scientific Institute and University Hospital San Raffaele, Milan, Italy ${ }^{7}$ Unitat de Neuroimmunologia Clinica and Magnetic Resonance Unit, Hospital Universitario Vall d'Hebron, Barcelona, Spain ${ }^{8}$ Multiple Sclerosis Center, Scientific Institute Santa Maria Nascente, Fondazione Don Gnocchi, Milan, Italy

\section{Correspondence to}

Dr 0 Ciccarelli, Department of Brain Repair and Rehabilitation, Institute of Neurology, Queen Square, London WC1N 3BG, UK; o.ciccarelli@ion.ucl.ac.uk

Received 30 November 2009 Revised 18 February 2010 Accepted 21 March 2010 Published Online First 13 July 2010

\section{UNLOCKID}

This paper is freely available online under the BMJ Journals unlocked scheme, see http:// jnnp.bmj.com/site/about/ unlocked.xhtml

\begin{abstract}
Objectives Prediction of long term clinical outcome in patients with primary progressive multiple sclerosis (PPMS) using imaging has important clinical implications, but remains challenging. We aimed to determine whether spatial location of T2 and T1 brain lesions predicts clinical progression during a 10-year follow-up in PPMS.
\end{abstract}

Methods Lesion probability maps of the $\mathrm{T} 2$ and $\mathrm{T} 1$ brain lesions were generated using the baseline scans of 80 patients with PPMS who were clinically assessed at baseline and then after 1, 2, 5 and 10 years. For each patient, the time (in years) taken before bilateral support was required to walk (time to event (TTE)) was used as a measure of progression rate. The probability of each voxel being 'lesional' was correlated with TTE, adjusting for age, gender, disease duration, centre and spinal cord cross sectional area, using a multiple linear regression model. To identify the best, independent predictor of progression, a Cox regression model was used.

Results A significant correlation between a shorter TTE and a higher probability of a voxel being lesional on T2 scans was found in the bilateral corticospinal tract and superior longitudinal fasciculus, and in the right inferior fronto-occipital fasciculus $(p<0.05)$. The best predictor of progression rate was the T2 lesion load measured along the right inferior fronto-occipital fasciculus $(p=0.016$, hazard ratio $1.00652,95 \% \mathrm{Cl} 1.00121$ to 1.01186 ).

Conclusion Our results suggest that the location of $\mathrm{T} 2$ brain lesions in the motor and associative tracts is an important contributor to the progression of disability in PPMS, and is independent of spinal cord involvement.

\section{INTRODUCTION}

Prediction of long term clinical outcome in patients with primary progressive multiple sclerosis (PPMS) has important clinical implications, in both the design of treatment trials and in providing prognostic advice to individual patients. Whole brain T2 and T1 lesion loads, which are objective measures of visible tissue damage, have been studied as potential predictors of long term clinical outcome in a large cohort of PPMS patients who were followed-up for 10 years in five European MAGNIMS (MRI in MS) centres. ${ }^{1}$ Neither T2 nor T1 lesion load at study entry predicted clinical deterioration over 10 years. ${ }^{1}$ This is surprising, since T2 lesion volume has been shown to predict disability after 20 years in patients who presented with clinically isolated syndrome, ${ }^{2}$ and suggests differences in the mechanisms of tissue damage between relapsing-remitting and PPMS.
In searching for other features of T2 and T1 brain lesions which could predict long term outcome in PPMS, in the same paper we examined the short term increase in $\mathrm{T} 2$ and $\mathrm{T} 1$ lesion loads over 2 years. We found that this also failed to predict progression over 10 years, ${ }^{1}$ although it was more promising over 5 years. ${ }^{3}{ }^{4}$ This suggests that accumulation of visible lesions over a short time showed no advantage over total lesion load at study entry when it came to predicting progression at 10 years. Thus, predictors which are promising in short term follow-up studies may not be as compelling in long term studies.

Another, mostly unexplored, feature of lesions which may be relevant to predicting progression is their topographic distribution. Therefore, we aimed to investigate whether the location of T2 and T1 lesions at baseline predicts progression over 10 years, performing a retrospective study in the same cohort of PPMS patients. ${ }^{1}$ To explore lesion location, we employed the Lesion Probability Mapping (LPM) approach, that provides a voxel-wise, quantitative description of the topographic distribution of brain lesions. ${ }^{5}$ Since a possible limitation of previous studies which explored predictors of progression, is the use of step changes of Expanded Disability Status Scale (EDSS) as the outcome measure, ${ }^{6}$ we defined the time (in years) taken to require bilateral support in order to walk as a measure of the individual progression rate. We then performed a time to event (TTE) analysis and combined it with LPM. Although cervical cord cross sectional area at study entry did not predict clinical outcome at 10 years in this cohort of PPMS patients, ${ }^{1}$ spinal cord damage is thought to play an important role in determining disability in $\mathrm{PPMS}^{7-9}$; therefore, we corrected our analysis for baseline cord cross-sectional area. We also tested whether regional T2 and T1 lesion loads located in specific, clinically strategic areas, were significant predictors of TTE.

\section{METHODS \\ Patients}

Of the 101 patients with PPMS who were followedup for 10 years in five European centres (Amsterdam, Barcelona, Bordeaux, London and Milan), ${ }^{1} 80$ participants, whose baseline imaging data were available, were included in this study. All of these patients were clinically assessed using the EDSS ${ }^{10}$ at baseline (see table 1 for baseline demographic, clinical and radiological characteristics), 76 after 1 year, 59 after 2 years, 75 after 5 years and 79 after 10 years. 
Table 1 Demographic, clinical and radiological characteristics of the patients studied in each centre, at study entry

\begin{tabular}{|c|c|c|c|c|c|}
\hline Characteristics & Amsterdam & Barcelona & Bordeaux & London & Milan \\
\hline Patients (n) & 20 & 22 & 4 & 22 & 12 \\
\hline Gender (F/M) & $10 / 10$ & $9 / 13$ & $3 / 1$ & $11 / 11$ & $2 / 10$ \\
\hline Age (years) (mean (range)) & $53.5(76-34)$ & $45.7(31-64)$ & $48.7(43-59)$ & $52.7(29-74)$ & $45.1(32-50)$ \\
\hline Disease duration (years) (mean (range)) & $11.7(2-32)$ & $8.8(3-24)$ & $9.0(2-11)$ & $10.3(1-24)$ & $6.8(2-17)$ \\
\hline EDSS score (median (range)) & $6.0(2-8)$ & $5.5(2-7.5)$ & $5.0(4-6)$ & $6.0(2.5-8.5)$ & $4.0(2-8.5)$ \\
\hline T2 lesion load (ml)(mean (SD)) & $8.4(11.0)$ & $15.8(16.0)$ & $12.6(17.7)$ & $15.3(17.5)$ & $7.7(5.7)$ \\
\hline T1 lesion load (ml)(mean (SD)) & $3.4(4.9)$ & $5.6(7.2)$ & $6.0(7.7)$ & $4.7(7.7)$ & $3.8(6.6)$ \\
\hline Spinal cord area $\left(\mathrm{mm}^{2}\right)$ (mean (SD)) & $72.3(10.1)$ & $77.9(8.2)$ & - & $70.3(9.4)$ & $77.4(8.1)$ \\
\hline
\end{tabular}

EDSS, Expanded Disability Status Scale.

Of the 79 patients assessed after 10 years, 36 were scored using the EDSS telephone interview. ${ }^{11}$ Four patients scored 10 on the EDSS as their death was due to MS; one death occurred between years 2 and 5 , and three between years 5 and 10 .

All subjects gave informed, written consent before the study, which was approved by the local Ethics Committees in each one of the participating centres.

\section{MRI acquisition}

Patients underwent the same imaging protocol in each centre at study entry. In London, data were acquired using a $1.5 \mathrm{~T}$ GE Signa scanner, whereas at the other four sites a $1.5 \mathrm{~T}$ Siemens scanner was used. The protocol included T1 and dual echo (T2 and proton density (PD) weighted spin echo) imaging of the brain (see supplementary table 1 online for the acquisition parameters in each centre). Volumetric images of the spinal cord were also acquired, as previously described. ${ }^{12}$

\section{MRI processing}

\section{Lesion probability map (LPM)}

For the following steps, we used the imaging tools available in the Functional MRI of the Brain's Software Library (FSL, http:// www.fmrib.ox.ac.uk/fsl).

1. Lesion masks

A single observer (VS), who was blinded to the clinical details, contoured the PD hyperintense and T1 hypointense lesions, with reference to the co-registered T2 weighted images. ${ }^{13}$ Binary lesion masks were then produced and individual lesion loads calculated.

2. Template construction

Each patient's T1 scan was registered to the Montreal Neurological Institute (MNI152) template using a fully affine transformation (12 parameters) (FLIRT - FMRIB's Linear Image Registration Tool), as per previous work in patient groups with a variety of neurological conditions. ${ }^{14-16}$ All of the the resulting transformed images were averaged to obtain the internal T1 template.

3. Transformation of individual scans and lesion masks into T1 template

Each patient's PD weighted scan was registered to the corresponding $\mathrm{T} 1$ scan in native space using a rigid body transformation and trilinear interpolation (with FLIRT). The transformation parameters were then applied to the T2 lesion masks, bringing them into alignment with the individual T1 scans. Each patient's T1 scan was then registered to the T1 template, using non-linear registration (FNIRT -FMRIB's Non Linear Image Registration Tool), and the resulting transformation parameters were applied to the T1 and T2 lesion masks, which had been previously registered onto the individual $\mathrm{T} 1$, using trilinear interpolation. In order to maintain the volume of the transformed lesion masks as close as possible to those in the native brain images, after the trilinear interpolation, the lesion masks were thresholded using a value of 0.5 . Two observers independently checked all the co-registered lesions masks ( $\mathrm{BB}, \mathrm{MB})$.

4. T2 and T1 LPMs

T2 and T1 LPMs were generated by averaging the T2 and T1 lesion masks, at each voxel, in standard space. For each map, the resulting voxel intensity indicates how frequently the voxel in question is within a lesion across all patients, ie, the probability of that voxel being lesional. Regions containing the peaks of lesion probability were localised using the Johns Hopkins University white matter tractography atlas, provided by FSL. ${ }^{17}$

Since male gender has been associated, in this patient cohort, with a worse long term outcome, ${ }^{1}$ the differences in the probability of each voxel being lesional between male and female patients were assessed using an unpaired t-test and corrected for multiple comparisons at cluster level (t 2, $\mathrm{p}<0.05)$ using permutation based inference. ${ }^{18}$

\section{Cord cross-sectional area}

Cord cross-sectional area at the C2-3 level was measured using a semiautomated technique previously described. ${ }^{19}$ Previous work has suggested that C2-3 level offers several anatomical advantages for measuring cord cross-sectional area, with high cord to cerebrospinal fluid contrast, limited inter-subject variability in cord cross-sectional area at this level and a low probability of disc protrusions.

\section{Statistical analysis}

1. Correlation between lesion location and time to event For each patient, the time (in years) taken from disease onset to reach the level of disability that requires a constant bilateral support to walk (ie, EDSS=6.5) was defined as the TTE and was used as a measure of progression rate. An EDSS of 6.5 was chosen as it is easy to recognise and represents a clinically meaningful stage in the progression of disability. It was also appropriate for our cohort, whose median EDSS at study entry was 5.7 (range 2-8.5). In cases where the EDSS was scored as equal or greater than 6.5 at a follow-up visit for the first time, it was assumed that the patient reached this score midway between the date of the visit and the preceding assessment. Patients were divided into three groups of similar size on the basis of their TTE: (1) patients who reached EDSS 6.5 before entering the study $(n=23)$; (2) patients who reached EDSS 6.5 between the baseline assessment and year $5(n=23)$; and (3) patients who reached EDSS 6.5 between year 5 and year 10 $(n=13)$ and those who had not reached the event at the end of the study $(n=21)$.

To identify regions where the probability of each voxel being lesional in T2-LPM and T1-LPM correlated with TTE, an 
ordinal multiple regression was used, with an indicator of the three TTE categories as regressor, and age, gender, disease duration at baseline, centre and cord cross-sectional area as additional covariates. Corrections for multiple comparisons at cluster level ( $\mathrm{t} 2, \mathrm{p}<0.05$ ) were performed using permutationbased inference. ${ }^{18}$

2. Predictors of progression rate

A Cox regression analysis was performed, entering TTE as the dependent variable, and the number of lesional voxels (or regional lesion loads) extracted from tracts that encompassed regions showing significant associations in the previous analysis, as independent variables. T2 and T1 total lesion loads, cord cross-sectional area, gender, age, disease duration and centre were entered as additional, independent variables. In particular, each independent variable was entered individually into the model. Subsequently, significant and borderline predictors were modelled together to determine the best, independent predictor. This analysis was performed using Stata 9.2 (http://www.stata. com) (Stata-Corp, College Station, Texas, USA); results with $\mathrm{p}<0.05$ were considered significant.

\section{RESULTS}

\section{T2 and T1 lesion probability maps}

The brain locations that showed the highest probability of detecting T2 hyperintense and T1 hypointense lesions were the superior and posterior regions of the corona radiata (figure 1). The maximum local probability was higher in T2-LPM compared with T1-LPM (42\% vs $26 \%$ ). No significant differences in the probability of a voxel being lesional in T2-LPM and T1LPM between men and women were found.

\section{Correlation between lesion location and time to event}

Of the 80 patients included in the study, 59 (73.7\%) reached the event (EDSS=6.5) either before entering the study (23 patients, $28.7 \%$ ) or during the course of the study (36 patients, $45 \%$ ), while $21(26.3 \%)$ had not reached the event when the study terminated. The median of the TTE calculated on those who reached EDSS 6.5, was 11.5 years (range 3-29 years).

There was a significant correlation between a higher probability of a voxel being lesional on T2-LPM and a shorter TTE $(p<0.05)$ in the following three regions: the bilateral corticospinal tract (CST) (from the cortex to the corona radiata), the bilateral superior longitudinal fasciculus (SLF) and the right inferior fronto-occipital fasciculus (IFOF) (figure 2). Conversely, there was no correlation between lesion probability on T1-LPMs and TTE, although a statistical trend towards a significant association was found in a region in the right posterior corona radiata which included the CST and the SLF $(p<0.08)$.

\section{Predictors of progression rate}

The number of lesional voxels and corresponding regional lesion loads extracted from tracts containing regions that showed an association between lesion location and TTE are summarised in table 2 .

When predictors were entered individually into the Cox regression model, we found that a greater number of T2 lesional voxels extracted from the right IFOF was associated with a shorter TTE $(p=0.01$, hazard ratio (HR) 1.00689 , 95\% CI 1.00158 to 1.01225 ); for every extra $\mathrm{ml}$ of lesions within the right IFOF, the time taken to reach EDSS 6.5 was halved. The number of T2 lesional voxels extracted from the bilateral CST
Figure 1 T2-Lesion probability map (LPM) (top row) and T1-LPM (bottom row); the colour code indicates the probability of a voxel being lesional. The maximum probability was found in the superior and posterior regions of the corona radiata, and was higher in T2LPM compared with T1-LPM (42\% vs $26 \%)$.

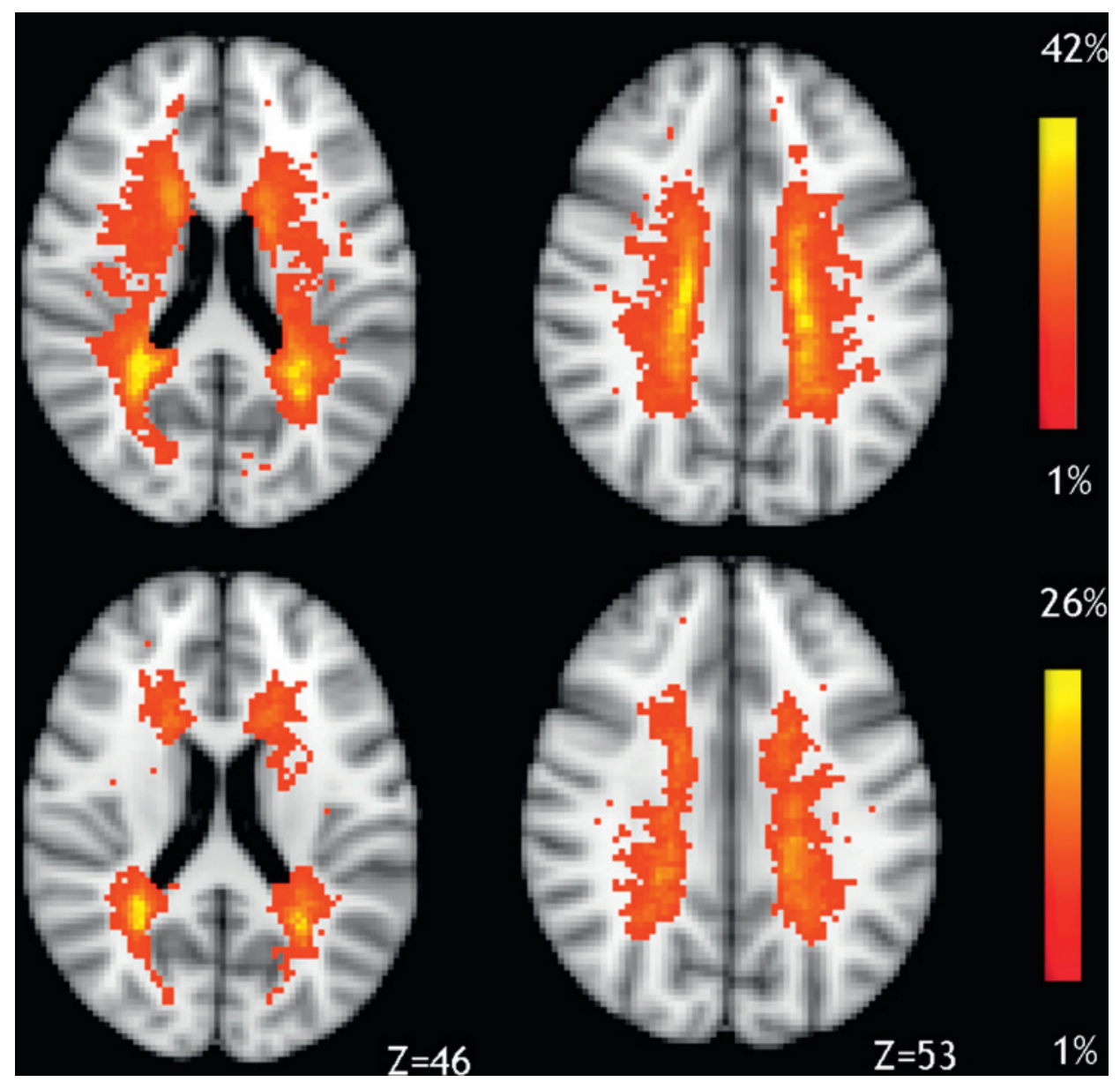


Figure 2 Red-orange voxels show the regions on the regression maps where a higher probability of a voxel being lesional on T2 images was significantly associated with a shorter time to event. These regions are: the bilateral corticospinal tract (CST) (from the cortex to the corona radiata), the bilateral superior longitudinal fasciculus (SLF) and the right inferior frontooccipital fasciculus (IFOF). In blue, yellow and green are displayed the courses of the three white matter tracts (bilateral CST, bilateral SLF and right IFOF, respectively).

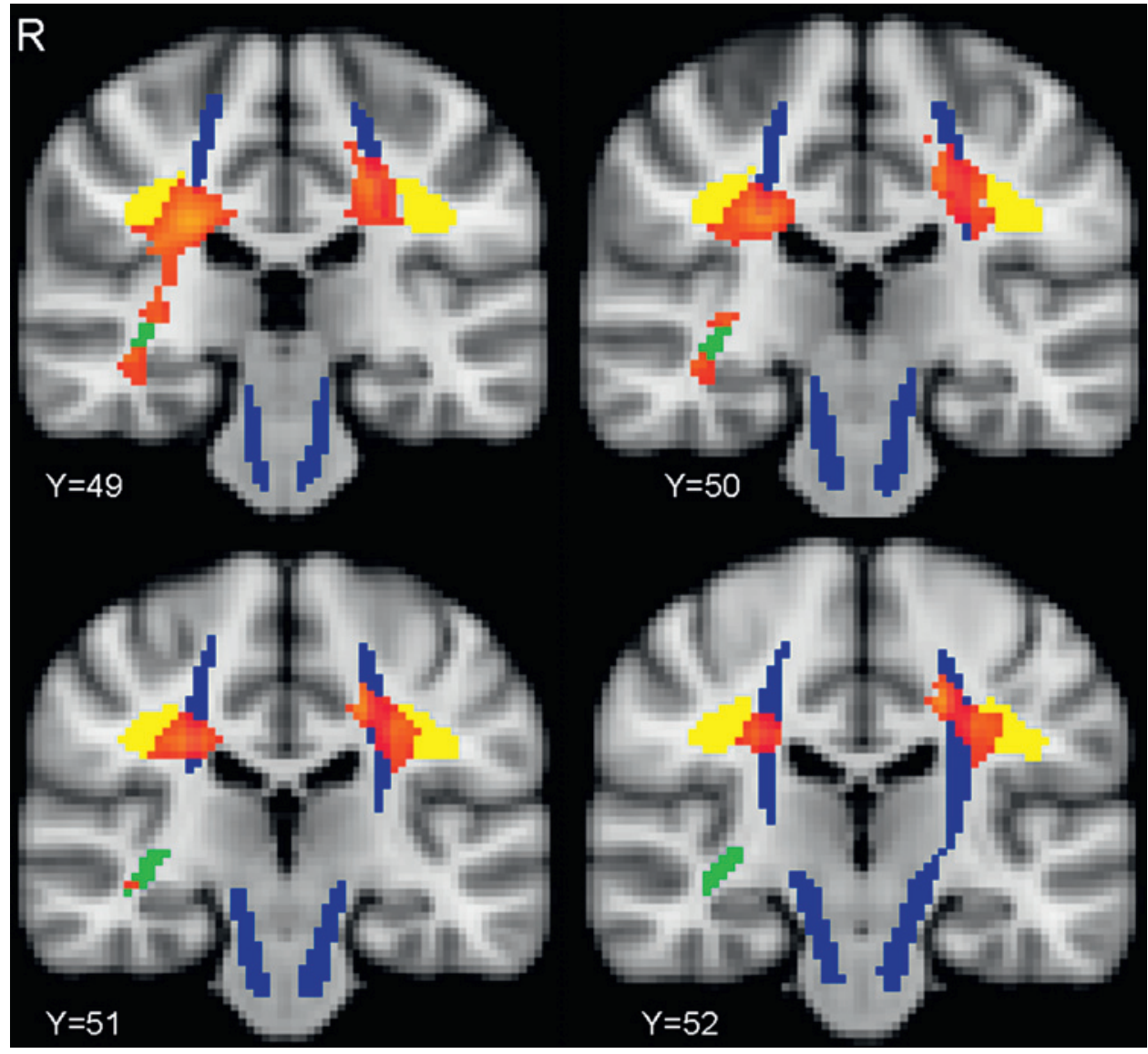

showed a trend towards a correlation with TTE $(p=0.07, \mathrm{HR}$ $1.002,95 \%$ CI 0.99981 to 1.00442 ); for every extra $\mathrm{ml}$ of lesions localised within the CSTs, the time taken to reach the event was reduced by $30 \%$. The number of lesional voxels in the bilateral SLF did not predict TTE.

Among the other variables tested individually in the Cox regression, we found that: (i) the whole brain T1 hypointense lesion load was significantly associated with TTE ( $p=0.04$, HR $1.00004,95 \%$ CI 1.000001 to 1.00007); for every extra $\mathrm{ml}$ in total $\mathrm{T} 1$ lesion load, the time taken to reach the event was reduced by $0.5 \%$; and (ii) there was a borderline significant association between gender and TTE $(p=0.062$, HR 1.62, 95\% CI 0.96 to $2.73)$; the time taken for men to reach the event was reduced by $60 \%$.

When all the significant and borderline predictors were entered together into the Cox regression model, the number of T2 lesional voxels in the right IFOF was the best independent predictor of TTE ( $p=0.016$, HR 1.00652, 95\% CI 1.00121 to 1.01186). The number of T2 lesional voxels in the bilateral CST ( $p=0.07$, HR 1.0022, 95\% CI 0.99979 to 1.00461$)$ and the whole brain T1 hypointense lesion load ( $p=0.07$, HR 1.00003, 95\% CI

Table 2 Number of lesional voxels and corresponding regional lesion loads extracted from the three tracts containing regions that showed an association between lesion location and time to event

\begin{tabular}{lccc}
\hline $\begin{array}{l}\text { Measures of regional } \\
\text { lesional damage }\end{array}$ & Bilateral CST & Bilateral SLF & Right IFOF \\
\hline No of lesional voxels (mean (SD)) & $37.6(58.8)$ & $38.4(76.1)$ & $30.1(47.4)$ \\
Lesion load (ml) (mean (SD)) & $0.3(0.5)$ & $0.3(0.6)$ & $0.2(0.4)$
\end{tabular}

CST, corticospinal tract; IFOF, inferior fronto-occipital fasciculus; SLF, superior longitudinal fasciculus.
0.99999 to 1.00006 ) became borderline independent predictors while gender became non-significant.

\section{DISCUSSION}

We found that the location of T2 lesions at baseline is associated with the rate of progression in PPMS over a 10 year period. In particular, a relationship between a higher probability of a voxel being lesional and a faster progression rate was found in the motor tract (ie, the bilateral CST) and two associative tracts (ie, the bilateral SLF and the right IFOF). These results extend the findings of previous cross-sectional studies that have found correlations between mobility and regional MRI abnormalities localised within the motor tract. ${ }^{20} 21$ Furthermore, the SLF and the IFOF are two associative tracts, which are part of the longitudinal association fibre system that connects each frontal lobe with other regions in the same hemisphere. ${ }^{22}$ They seem to be involved with high order aspects of motor function, as shown in primate studies. ${ }^{23}$ Therefore, a possible interpretation of our findings is that disruption of long associative tracts, due to the presence of lesions, causes a disconnection syndrome that may contribute to a more rapid deterioration of mobility. ${ }^{24}$ In fact, it is known that disrupted white matter pathways, which play a crucial role in the coordination of information flow between different grey matter regions, are likely to lead to an altered functional connectivity between regions and, ultimately, to clinical impairment. ${ }^{25}$ Furthermore, damage in these tracts may reduce the cortical reorganisation that originates from activation of regions connected through these pathways, thus resulting in a reduced ability of the brain to limit the clinical impact of structural damage. We found that the relationship between lesion location in the IFOF and TTE was statistically significant on the right side only. This is in agreement with the reports of 
asymmetry in MS brain pathology. ${ }^{26}$ In future, the contribution of handedness to these results needs to be explored.

The association between a higher probability of a voxel being lesional and a faster progression rate, was independent of spinal cord atrophy (the mean value of cord cross-sectional area in patients is similar to that previously reported in PPMS). ${ }^{8}$ Interestingly, when the correlation between lesion location and TTE was investigated using both EDSS equal to 6 and EDSS equal to 7 as events, the same motor and associative tracts showed significance (results not shown), suggesting that the location of T2 brain lesions in these tracts is an important factor in contributing to disability progression in PPMS.

When we investigated the best predictors of TTE, we found that the regional T2 lesion volume measured in the right IFOF was the best, independent predictor of progression. Conversely, the T2 lesion load in the bilateral CST became a borderline predictor, and that in the SLF became non-significant, suggesting a preferential role of the right IFOF, perhaps through the inferoand dorso-lateral frontal, and occipital cortices that it connects. ${ }^{22}$ Indeed, the IFOF constitutes the inferior part of the fronto-occipital fasciculus, that is thought to be involved in the use of visual information for the purpose of guiding movements and controlling motor actions. ${ }^{27}$

As previously demonstrated, the whole brain T2 lesion load did not correlate significantly with the progression rate, supporting the concept that in MS a proportion of the total lesion load is clinically silent ${ }^{15} 28-30$ and is not as relevant in determining progression as other lesions localised in strategic areas.

In contrast with our data about T2 lesions, we did not find any significant association between the $\mathrm{T} 1$ hypointense lesion probability and progression rate. A possible interpretation for this finding is that the T1 lesion load is considerably smaller than the T2 lesion load and, therefore, tracts are less likely to contain T1 compared with T2 lesions. This, in turn, means that the range of lesion loads within a given region is smaller for T1 compared with T2 lesions, reducing the possibility of a correlation with clinical outcomes. Interestingly, we found that whole brain T1 lesion load was a borderline independent predictor of progression rate. This is not surprising, since T1 hypointense lesions are known to be histopathologically associated with severe tissue destruction ${ }^{31}$ and T1 total lesion load correlates with clinical disability in MS more strongly than T2 lesion load. ${ }^{32} 33$

We found that the areas most likely to contain lesions were similar in the T2-LPMs and T1-LPMs, and included the superior and posterior regions of the corona radiata. This is interesting, since a large number of axonal projections that contribute to the generation of the CST converge in this region, ${ }^{34}$ and PPMS is typically characterised by a progressive decline in motor function. A previous study reported that patients with PPMS had a greater probability of a lesion occurring in these regions, compared with relapsing-remitting patients. ${ }^{5}$

An interesting aspect of this study is that we performed a TTE analysis which, in MS, has been limited to clinical trials. ${ }^{35} 36$ In fact, TTE analysis can be easily adapted to different events as long as the event in question is temporally defined and clinically meaningful. ${ }^{37}$ When the event cannot be specifically dated, it is acceptable to use the midpoint between the visit at which the event is noted and the previous one, as the time of the event. ${ }^{38}$ However, due to our study design, the interval between visits varied between 1 and 5 years, and 13 patients (16\%) reached the event during the 5 years which elapsed between the last two time points. When we repeated the analysis without these patients, we found that the results were very similar to those presented here (results not shown), and so concluded that the variability in follow-up intervals has not significantly distorted the results. Furthermore, the progression rate that we observed in our population was similar to the one previously reported in epidemiological studies in PPMS patients, ${ }^{39}$ suggesting that despite the retrospective design of the study and the suboptimal frequency of assessments, the definition of the clinical outcome was appropriate.

We have studied a large number of PPMS patients over a very long follow-up period, recruited in five European centres. Another potential limitation of this study is that, with the available data, we were not able to look for grey matter lesions 4041 that may be very relevant in contributing to long term clinical disability. Another possible limitation of this study is the potential for registration errors and consequent lesion location inaccuracies related to the LPM technique. Two observers, rather than one, ${ }^{5} 15$ inspected registered lesion masks and were in agreement in all cases. While it is to be expected that there will be some residual misalignments, in the context of a lesion probability analysis, the effects of this will be negligible.

In conclusion, this study has provided insights into the mechanisms of progression in PPMS, reporting that T2 lesion location at study entry is a major determinant of clinical progression independent of spinal cord atrophy. Furthermore, we have confirmed that the time to bilateral support is a useful approach to identify those who progress more rapidly.

Acknowledgements The authors are very grateful to Dr Valerie Stevenson for her analysis of the baseline scans and to all of the patients for participating in the study

Funding BB is funded by FISM (Fondazione Italiana Sclerosi Multipla - 2007/B/01). OC is a Wellcome Trust Advanced Fellow. This work was undertaken at UCLH/UCL who received a proportion of funding from the Department of Health's NIHR Biomedical Research Centres funding scheme.

\section{Competing interests None.}

Ethics approval This study was approved by the local ethics committees in each one of the participating centres (Amsterdam, Barcelona, Bordeaux, London and Milan)

Provenance and peer review Not commissioned; externally peer reviewed.

\section{REFERENCES}

1. Khaleeli Z, Ciccarelli O, Manfredonia F, et al. Predicting progression in primary progressive multiple sclerosis: a 10-year multicenter study. Ann.Neuro/ 2008:63:790-3.

2. Fisniku LK, Brex PA, Altmann DR, et al. Disability and T2 MRI lesions: a 20-year followup of patients with relapse onset of multiple sclerosis. Brain 2008;131:808-17.

3. Ingle GT, Stevenson VL, Miller DH, et al. Primary progressive multiple sclerosis: a 5-year clinical and MR study. Brain 2003;126:2528-36.

4. Sastre-Garriga J, Ingle GT, Rovaris M, et al. Long-term clinical outcome of primary progressive MS: predictive value of clinical and MRI data. Neurology 2005:65:633-5.

5. Di Perri C, Battaglini M, Stromillo ML, et al. Voxel-based assessment of differences in damage and distribution of white matter lesions between patients with primary progressive and relapsing-remitting multiple sclerosis. Arch Neurol 2008;65:236-43.

6. Kragt JJ, Thompson AJ, Montalban X, et al. Responsiveness and predictive value of EDSS and MSFC in primary progressive MS. Neurology 2008;70:1084-91.

7. Nijeholt GJ, van Walderveen MA, Castelijns JA, et al. Brain and spinal cord abnormalities in multiple sclerosis. Correlation between MRI parameters, clinical subtypes and symptoms. Brain 1998;121(Pt 4):687-97.

8. Rovaris M, Bozzali M, Santuccio G, et al. In vivo assessment of the brain and cervical cord pathology of patients with primary progressive multiple sclerosis. Brain 2001;124:2540-9.

9. Bieniek M, Altmann DR, Davies GR, et al. Cord atrophy separates early primary progressive and relapsing remitting multiple sclerosis. J Neurol Neurosurg Psychiatry 2006; 77:1036-9

10. Kurtzke JF. Rating neurologic impairment in multiple sclerosis: an expanded disability status scale (EDSS). Neurology 1983;33:1444-52

11. Lechner-Scott J, Kappos L, Hofman M, et al. Can the Expanded Disability Status Scale be assessed by telephone? Mult Scler 2003;9:154-9.

12. Stevenson VL, Miller DH, Rovaris M, et al. Primary and transitional progressive MS a clinical and MRI cross-sectional study. Neurology 1999;52:839-45.

13. Plummer D. Displmage: a display and analysis tool for medical images. Rev Neuroradiol 1992;5:489-95.

14. Ginestroni A, Battaglini M, Della Nave R, et al. Early structural changes in individuals at risk of familial Alzheimer's disease: a volumetry and magnetization transfer MR imaging study. J Neurol 2009;256:925-32. 
15. Vellinga MM, Geurts JJ, Rostrup E, et al. Clinical correlations of brain lesion distribution in multiple sclerosis. J Magn Reson Imaging 2009;29:768-73.

16. Karagulle Kendi AT, Lehericy S, Luciana M, et al. Altered diffusion in the frontal lobe in Parkinson disease. AJNR Am J Neuroradiol 2008;29:501-5.

17. Mori S, Wakana S, Nagae-Poetscher LM, et al. MRI atlas of human white matter Amsterdam, The Netherlands: Elsevier, 2005.

18. Nichols TE, Holmes AP. Nonparametric permutation tests for functional neuroimaging: a primer with examples. Hum Brain Mapp 2002;15:1-25

19. Losseff NA, Webb SL, O'Riordan Jl, et al. Spinal cord atrophy and disability in multiple sclerosis. A new reproducible and sensitive MRI method with potential to monitor disease progression. Brain 1996;119(Pt 3):701-8.

20. Wilson M, Tench CR, Morgan PS, et al. Pyramidal tract mapping by diffusion tensor magnetic resonance imaging in multiple sclerosis: improving correlations with disability. J Neurol Neurosurg Psychiatry 2003;74:203-7.

21. Reich DS, Zackowski KM, Gordon-Lipkin EM, et al. Corticospinal tract abnormalities are associated with weakness in multiple sclerosis. AJNR Am J Neuroradiol 2008:29:333-9.

22. Catani M, Howard RJ, Pajevic $\mathrm{S}$, et al. Virtual in vivo interactive dissection of white matter fasciculi in the human brain. Neuroimage 2002;17:77-94.

23. Rizzolatti G, Matelli M. Two different streams form the dorsal visual system anatomy and functions. Exp Brain Res 2003;153:146-57.

24. He Y, Dagher A, Chen Z, et al. Impaired small-world efficiency in structural cortical networks in multiple sclerosis associated with white matter lesion load. Brain 2009; 132:3366-79.

25. Rocca MA, Colombo B, Falini A, et al. Cortical adaptation in patients with MS: a cross-sectional functional MRI study of disease phenotypes. Lancet Neurol 2005;4:618-26

26. Reich DS, Smith SA, Zackowski KM, et al. Multiparametric magnetic resonance imaging analysis of the corticospinal tract in multiple sclerosis. Neuroimage 2007; 38:271-9.

27. Schmahmann JD, Pandya DN. The complex history of the fronto-occipital fasciculus. J Hist Neurosci 2007;16:362-77.

28. Li DK, Held U, Petkau J, et al. MRI T2 lesion burden in multiple sclerosis: a plateauing relationship with clinical disability. Neurology 2006;66:1384-9.
29. Miki Y, Grossman Rl, Udupa JK, et al. Relapsing-remitting multiple sclerosis: longitudinal analysis of MR images-lack of correlation between changes in T2 lesion volume and clinical findings. Radiology 1999;213:395-9.

30. Rudick RA, Lee JC, Simon J, et al. Significance of T2 lesions in multiple sclerosis: a 13-year longitudinal study. Ann Neurol 2006;60:236-42.

31. van Walderveen MA, Kamphorst W, Scheltens P, et al. Histopathologic correlate of hypointense lesions on T1-weighted spin-echo MRI in multiple sclerosis. Neurology 1998:50:1282-8.

32. Truyen $\mathrm{L}$, van Waesberghe $\mathrm{JH}$, van Walderveen MA, et al. Accumulation of hypointense lesions ("black holes") on T1 spin-echo MRI correlates with disease progression in multiple sclerosis. Neurology 1996;47:1469-76.

33. O'Riordan JI, Gawne CM, Coles A, et al. T1 hypointense lesion load in secondary progressive multiple sclerosis: a comparison of pre versus post contrast loads and of manual versus semi automated threshold techniques for lesion segmentation. Mult Scler 1998:4:408-12

34. Wakana S, Jiang H, Nagae-Poetscher LM, et al. Fiber tract-based atlas of human white matter anatomy. Radiology 2004:230:77-87.

35. Panitch H, Miller A, Paty D, et al. Interferon beta-1b in secondary progressive MS: results from a 3-year controlled study. Neurology 2004;63:1788-95.

36. Montalban X, Sastre-Garriga J, Tintore M, et al. A single-centre, randomized, double-blind, placebo-controlled study of interferon beta-1b on primary progressive and transitional multiple sclerosis. Mult Scler 2009;15:1195-205.

37. Allen C, Jiang K, Malbecq W, et al. Time-to-event analysis, or who gets better sooner? An emerging concept in headache study methodology. Cephalalgia 1999;19:552-6.

38. Law CG, Brookmeyer R. Effects of mid-point imputation on the analysis of doubly censored data. Stat Med 1992:11:1569-78.

39. Cottrell DA, Kremenchutzky M, Rice GP, et al. The natural history of multiple sclerosis: a geographically based study. 5. The clinical features and natural history of primary progressive multiple sclerosis. Brain 1999;122(Pt 4):625-39.

40. Kidd D, Barkhof F, McConnell R, et al. Cortical lesions in multiple sclerosis. Brain 1999:122(Pt 1):17-26.

41. Geurts JJ, Bo L, Pouwels PJ, et al. Cortical lesions in multiple sclerosis: combined postmortem MR imaging and histopathology. AJNR Am J Neuroradiol 2005:26:572-7. 